\title{
On the final size of epidemics with seasonality
}

\author{
Nicolas BACAËR* M. Gabriela M. GOMES ${ }^{\ddagger}$
}

\begin{abstract}
We first study an SIR system of differential equations with periodic coefficients describing an epidemic in a seasonal environment. Unlike in a constant environment, the final epidemic size may not be an increasing function of the basic reproduction number $\mathcal{R}_{0}$ or of the initial fraction of infected people. Moreover, large epidemics can happen even if $\mathcal{R}_{0}<1$. But like in a constant environment, the final epidemic size tends to 0 when $\mathcal{R}_{0}<1$ and the initial fraction of infected people tends to 0 . When $\mathcal{R}_{0}>1$, the final epidemic size is bigger than the fraction $1-1 / \mathcal{R}_{0}$ of the initially nonimmune population. In summary, the basic reproduction number $\mathcal{R}_{0}$ keeps its classical threshold property but many other properties are no longer true in a seasonal environment. These theoretical results should be kept in mind when analyzing data for emerging vector-borne diseases (West-Nile, dengue, chikungunya) or air-borne diseases (SARS, pandemic influenza), all these diseases being influenced by seasonality.
\end{abstract}

Keywords: basic reproduction number, seasonality, final epidemic size.

\section{Introduction}

Consider the following SIR system describing an epidemic

$$
\frac{d S}{d t}=-\beta(t) S I, \quad \frac{d I}{d t}=\beta(t) S I-\gamma(t) I, \quad \frac{d R}{d t}=\gamma(t) I,
$$

with continuous positive $\tau$-periodic contact rate $\beta(t)$ and recovery rate $\gamma(t)$. The function $S(t)$ is the fraction of the population that is susceptible (i.e., not yet infected), $I(t)$ the fraction that is infected, $R(t)$ the fraction that has recovered from infection and become immune, so that $S(t)+I(t)+R(t)=1$. Consider the initial condition

$$
S\left(t_{0}\right)=1-i-r, \quad I\left(t_{0}\right)=i, \quad R\left(t_{0}\right)=r,
$$

\footnotetext{
* Corresponding author. IRD (Institut de Recherche pour le Développement), 32 avenue Henri Varagnat, 93413 Bondy, France. E-mail: bacaer@bondy.ird.fr

${ }^{\dagger}$ Instituto Gulbenkian de Ciência, Apartado 14, 2781-901 Oeiras, Portugal.

${ }^{\ddagger}$ Centro de Matemática e Aplicações Fundamentais, Universidade de Lisboa, Av. Prof. Gama Pinto 2, 1649-003 Lisboa, Portugal.
} 
with $i>0, r \geq 0$ and $i+r<1$. Notice that the trivial cases $i=0$ and $i+r=1$ are excluded and that the special case $r=0$ corresponds to emerging diseases for which the population has no immunity. Let $R^{*}$ be the limit of $R(t)$ as $t \rightarrow+\infty$. Then $R^{*}-r$ is the final epidemic size. $R^{*}$ depends on the functions $\beta(t)$ and $\gamma(t)$ and on the parameters $t_{0}, i$ and $r$. To emphasize this dependence, we could write $R^{*}=R^{*}\left(\beta(\cdot), \gamma(\cdot), t_{0}, i, r\right)$. System (1) with a periodic $\beta(t)$ and a constant $\gamma$ can be used for epidemics of viral air-borne diseases that occur on a fast time scale compared to demographic processes and to the immune period, such as influenza (flu) and SARS.

When $\beta(t)$ and $\gamma(t)$ are both constant, (1) is the "simplified KermackMcKendrick system" $[1,2]$. In this special case, there is a closed but implicit formula for $R^{*}$ :

$$
\left(1-R^{*}\right) \exp \left[\mathcal{R}_{0} \frac{R^{*}-r}{1-r}\right]=1-i-r,
$$

where $\mathcal{R}_{0}=\beta / \gamma$ is the "basic reproduction number". It follows that $R^{*}$ is an increasing function of $\mathcal{R}_{0}$, that it is independent of $t_{0}$, and that it is an increasing function of $i$. All these properties are quite intuitive. If $\mathcal{R}_{0}<1$ then $R^{*} \rightarrow r$ when $i \rightarrow 0$. If $\mathcal{R}_{0}>1$ then

$$
R^{*}-r \geq(1-r)\left(1-1 / \mathcal{R}_{0}\right),
$$

as is easily checked by studying the left side of (3) as a function of $R^{*}$ (see also [2, Theor. 18.6]). $R^{*}$ converges when $i \rightarrow 0$ to a positive limit if $\mathcal{R}_{0}>1$. Assuming $r=0$ (emerging disease), this limit can be identified with the result of a post-epidemic seroprevalence survey. Then (3) gives an estimate of $\mathcal{R}_{0}$, which in turn can give an estimate of the vaccination coverage necessary to prevent an epidemic of the same disease in other areas with similar characteristics.

The problem of the definition of the basic reproduction number for periodic systems has been investigated in $[3,4,5,6,7]$. In summary, we have for system (1)

$$
\mathcal{R}_{0}=\frac{\bar{\beta}(1-r)}{\bar{\gamma}}, \quad \bar{\beta}=\frac{1}{\tau} \int_{0}^{\tau} \beta(t) d t, \quad \bar{\gamma}=\frac{1}{\tau} \int_{0}^{\tau} \gamma(t) d t .
$$

Indeed, linearizing (1) near the disease-free steady state $(S=1-r, I=0, R=r)$, we see that $d I / d t \simeq \beta(t)(1-r) I-\gamma(t) I . \mathcal{R}_{0}=1$ is obviously a threshold for this simple linear periodic equation. But one can also show that $\mathcal{R}_{0}$ is the spectral radius of the next-generation integral operator acting on periodic continuous functions

$$
\phi(t) \longmapsto \int_{0}^{\infty} K(t, x) \phi(t-x) d x,
$$

where $K(t, x)=\beta(t)(1-r) \exp \left(-\int_{t-x}^{t} \gamma(s) d s\right)$ is the rate of secondary cases produced at time $t$ by somebody infected at time $t-x[3, \S 5]$. This approach is close to the "usual" definition of $\mathcal{R}_{0}$ in a constant environment as the average number of secondary cases produced by an initial case. But seasonality introduces a level of complexity similar to that in age-structured epidemic models, for which $\mathcal{R}_{0}$ is again the spectral radius of an integral operator [8]. It is also easily 
shown that $\mathcal{R}_{0}$ is the unique real positive number such that the linear periodic system $d I / d t=\beta(t)(1-r) I / \mathcal{R}_{0}-\gamma(t) I$ has a dominant Floquet multiplier equal to 1 (see $[4, \S 3.4]$ and [6]). $\mathcal{R}_{0}$ appears also in the analysis of periodic birth and death processes $[4, \S 5.2]$. Notice that we call $\mathcal{R}_{0}$ the basic reproduction number, while some authors would call it the effective reproduction number and keep $\mathcal{R}_{0}$ for the ratio $\bar{\beta} / \bar{\gamma}$. In any case, $\mathcal{R}_{0}$ does not depend on $i$ and $t_{0}$.

In Section 2 we start investigating which properties of the simplified KermackMcKendrick system remain true in the periodic variant (1). It turns out that $R^{*}$ may not be an increasing function of $\mathcal{R}_{0}$, that it is a $\tau$-periodic function of $t_{0}$, and that it may not be an increasing function of $i$. The first and third properties are somewhat counterintuitive. The first property implies that it may be impossible to estimate $\mathcal{R}_{0}$ from seroprevalence data. Simulations also show that large epidemics can occur even when $\mathcal{R}_{0}<1$. This happens if the disease is introduced during a favorable period, if the initial fraction of infected people is not too small, if seasonality is sufficiently marked, and if the average infectious period $1 / \gamma$ is short compared to the length $\tau$ of the season. The 2007 chikungunya epidemic in Italy may have been one such case [9]. One should not conclude that $\mathcal{R}_{0}>1$ just because an epidemic peak is observed and one should be careful at how $\mathcal{R}_{0}$ is defined if seasonality is believed to be important. Simulations also show that the final epidemic size can be very sensitive to small changes in $\mathcal{R}_{0}$. This may explain why it is so difficult to predict the future of epidemics influenced by seasonality, as noticed during the 2005-2006 chikungunya epidemic in Réunion (an island of the Indian ocean).

In section 3 we show that, as in the simplified Kermack-McKendrick system, $\mathcal{R}_{0}=1$ is a threshold for the nonlinear periodic system (1). More precisely, we show that

- if $\mathcal{R}_{0}<1$, then $R^{*}-r \rightarrow 0$ when $i \rightarrow 0$.

- if $\mathcal{R}_{0}>1$, then $R^{*}-r \geq(1-r)\left(1-1 / \mathcal{R}_{0}\right)$ for all $0<i<1-r$.

Notice that for the case $\mathcal{R}_{0}>1$, we have $1-R^{*} \leq(1-r) / \mathcal{R}_{0}$. So the epidemic divides the initial nonimmune population by a number greater than $\mathcal{R}_{0}$. In some sense, this is like classical vaccination theory for systems with constant coefficients [10]. Similar threshold theorems have been or can be derived for various generalizations of the simplified Kermack-McKendrick system $[1,2,8$, $10,11,12]$. But our method of proof will be different because we could not find any final-size equation similar to (3) for systems with periodic coefficients. In section 3 we also prove that the threshold theorem remains true for a periodic SEIR system and for a periodic system describing vector-borne diseases, $\mathcal{R}_{0}$ being most easily defined and computed as in $[4, \S 3.4]$ (see also [6]).

\section{Numerical simulations}

To keep things simple and because of the widespread interest in pandemic flu, we will use the periodic SIR system although the discussion will be extended to 
a vector-borne disease (chikungunya). One can check that similar qualitative remarks can be made using the system of $\S 3.3$. So let us consider (1) with for example $\beta(t)=\bar{\beta}(1+\varepsilon \sin 2 \pi t / \tau)$, where $\tau=1$ year represents seasonality and obviously cannot be changed. In this section, we shall assume that $r=0$ (emerging disease) and will investigate how $R^{*}$ depends on the other parameters: $\bar{\beta}, \varepsilon, \gamma, t_{0}$, and $i$.

Fig. 1a shows that the final epidemic size $R^{*}$ may not increase with the basic reproduction number $\mathcal{R}_{0}=\bar{\beta} / \gamma$. The parameter values are $\varepsilon=0.5$, $1 / \gamma=1$ week $=1 / 52$ year, $t_{0} / \tau=0.5, i=10^{-3}$, and we took two values for $\bar{\beta}$ corresponding to $\mathcal{R}_{0}=2$ and $\mathcal{R}_{0}=2.5$. With the higher value of $\mathcal{R}_{0}$, the epidemic occurs during the unfavorable season $0.5<t / \tau<1$, when $\beta(t)$ is below its average. When the favorable season arrives $(1<t / \tau<1.5)$, the stock of susceptibles has already been largely depleted so no new epidemic peak occurs. For the smaller value of $\mathcal{R}_{0}$, the stock of susceptibles has not been depleted enough, a second epidemic wave occurs and the final epidemic size is larger. The latter situation is precisely what happened in 2005-2006 in Réunion, a small island of the Indian ocean and a French overseas territory. A first small peak occured in May 2005, just before the beginning of "winter" in the southern hemisphere. The epidemic crossed the winter at a low level. A second big epidemic peak occured at the beginning of the next "summer" in January 2006 and infected about 250,000 people, one third of the island's population. Notice finally that if the final epidemic size $R^{*}$ is not a monotone increasing function of $\mathcal{R}_{0}$, then it is impossible to estimate $\mathcal{R}_{0}$ from $R^{*}$ and in particular from seroprevalence data. However, we will show in Section 3 that $R^{*}-r \geq$ $(1-r)\left(1-1 / \mathcal{R}_{0}\right)$. So we know at least that $\mathcal{R}_{0} \leq(1-r) /\left(1-R^{*}\right)$, which gives an upper bound for $\mathcal{R}_{0}$.

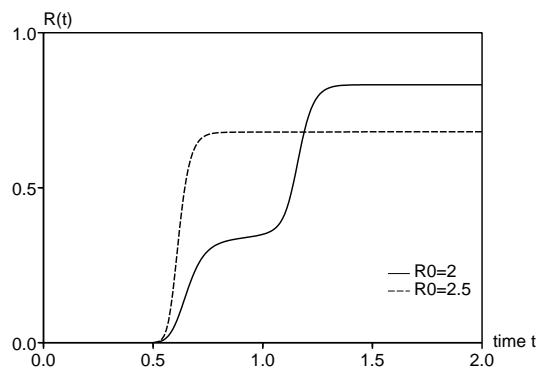

(a)

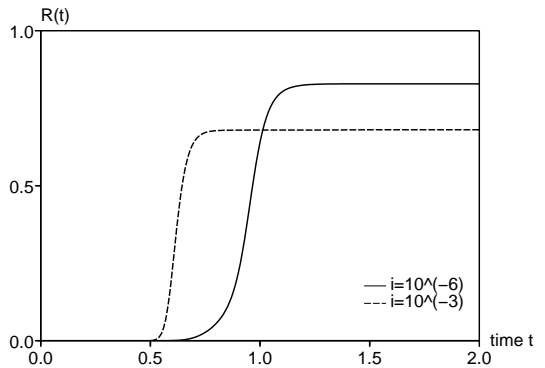

(b)

Figure 1: The final epidemic size may not increase with the basic reproduction number $\mathcal{R}_{0}$ (a) or with the initial fraction $i$ of infected people (b).

Similarly, Fig. 1b shows that the final epidemic size $R^{*}$ may not increase with the initial fraction $i$ of infected people. The parameter values are $\varepsilon=0.5$, $1 / \gamma=1 / 52$ year, $t_{0} / \tau=0.5, \mathcal{R}_{0}=2.5$ (which fixes $\bar{\beta}$ ), and we took either $i=10^{-6}$ or $i=10^{-3}$. Again, $i=10^{-6}$ depletes the number of susceptibles 
more slowly during the unfavorable season.

Fig. 2a shows that large epidemics are possible even if $\mathcal{R}_{0}<1$. The parameter values are $\mathcal{R}_{0}=0.9, \varepsilon=0.5,1 / \gamma=1 / 52$ year, $t_{0} / \tau=0$, and $i=10^{-3}$. The fact that $\mathcal{R}_{0}(1+\varepsilon)>1$ but $\mathcal{R}_{0}(1-\varepsilon)<1$ gives an indication of what is happening [more generally, (1) shows that $d I / d t<0$ when $\beta(t) / \gamma(t)<1$ ]. The epidemic occurs during the favorable season and simply stops when the unfavorable period arrives. The fact that the initial fraction of infected people is not too small $\left(i=10^{-3}\right)$ also plays a role. Indeed, the threshold theorem with $r=0$ shows that $R^{*} \rightarrow 0$ when $i \rightarrow 0$ and $\mathcal{R}_{0}<1$. From these remarks, one concludes that one should be careful before saying that $\mathcal{R}_{0}>1$ whenever an epidemic peak is observed. In the summer of 2007, a small chikungunya epidemic occured near Ravenna in Italy. Summer is the best season for mosquitoes in that area and the epidemic could probably never have crossed the winter. In our opinion, the estimates of $\mathcal{R}_{0}$ presented during the chikungunya modeling meeting at the European Centre for Disease Control and Prevention, which were all well above 1 , should be considered with caution [9]. The problem lies essentially in the definition of $\mathcal{R}_{0}$ and in the assumptions of the model. A model with a constant environment similar to the summer conditions cannot explain why the epidemic does not continue during falls, and is clearly inappropriate if the epidemic lasts two years as in Réunion.

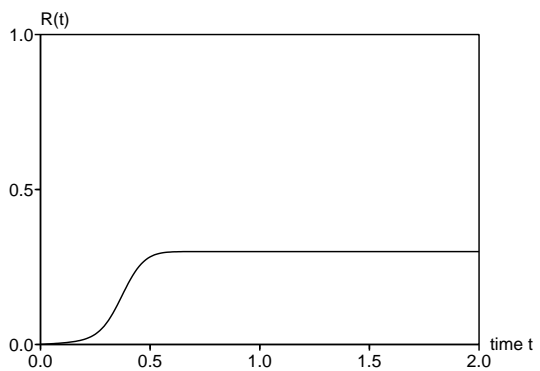

(a)

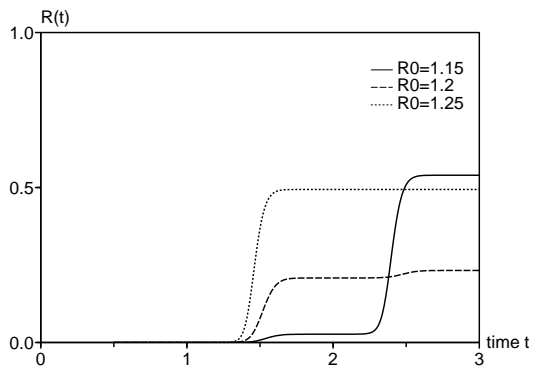

(b)

Figure 2: (a) Large epidemics can happen even if $\mathcal{R}_{0}<1$. (b) $R^{*}$ can be very sensitive to small changes in $\mathcal{R}_{0}$.

Fig. 2b shows that the final epidemic size $R^{*}$ can be very sensitive to small changes in $\mathcal{R}_{0}$. The parameters values are $\varepsilon=0.5,1 / \gamma=1 / 52$ year, $t_{0} / \tau=$ $0.5, i=10^{-6}$, while $\mathcal{R}_{0}$ can take one of the three values 1.15 (plain line), 1.2 (dashed line), and 1.25 (dotted line). We obtained $R^{*} \simeq 54 \%$ when $\mathcal{R}_{0}=1.15$, $R^{*} \simeq 23 \%$ when $\mathcal{R}_{0}=1.2$, and $R^{*} \simeq 50 \%$ when $\mathcal{R}_{0}=1.25$. In practice, one may never be able to distinguish values of $\mathcal{R}_{0}$, which are so close. But the corresponding final epidemic size varies by a factor 2 . In systems with periodic coefficients such as (1), forecasting the final epidemic size seems very difficult. This can answer in some way the critics directed against the epidemiologists, who followed the chikungunya epidemic in Réunion. Although the epidemic 
had been carefully monitored by a surveillance network since its beginning in April 2005, epidemiologists were not able to foresee the huge peak that occured in January and February 2006. This led to much pressure set by the general public and politicians on the "Institut de Veille Sanitaire", which is in charge of disease surveillance in France and its overseas territories. Our simulations suggest that this pressure may have been unjustified. In some way, epidemic forecasting beyond a few weeks in a seasonal environment may be almost as difficult as weather forecasting beyond a few days. Recall that the difficulty of analyzing endemic (not emerging) diseases in a seasonal environment, chaos often being suspected, is a somewhat different question as the one studied here.

For Fig. 2b, we chose $i=10^{-6}$. In practice, it is difficult to estimate the initial fraction $i$ of infected people. The problem is that the SIR system assumes some kind of homogeneous mixing. If an epidemic starts in a city from just one initial case, one may think that $i$ is simply one over the population of the city. But if the city is large, homogeneous mixing may not be reasonable and we may have to use the population of the quarter of the city where the initial case was introduced. The problem is the same for epidemics in a small island like Réunion but with a population of 800,000 , which is concentrated along the coast.

Fig. 3a investigates the dependence of the final epidemic size $R^{*}$ on the time $t_{0}$ at which the epidemic starts. Obviously, $R^{*}$ is always a $\tau$-periodic function of $t_{0}$, system (1) being invariant by a shift of $\tau$ in time. The parameter values in Fig. $3 \mathrm{a}$ are $\mathcal{R}_{0}=1$ or $\mathcal{R}_{0}=1.5, \varepsilon=0.5,1 / \gamma=1$ week or 3 weeks, and $i=10^{-3}$. The dependence on $t_{0}$ is strong if $\mathcal{R}_{0}$ is close to 1 and if the infectious period $1 / \gamma$ is short compared to the seasonal period $\tau$. In such a case, the epidemic cannot develop during the unfavorable season. Fig. 3b shows for $\mathcal{R}_{0}=1$ the "reproductive value" $V\left(t_{0}\right)$ ("infectious value" might be a more appropriate expression) of an initial case introduced at time $t_{0}$, computed from the linearized equation near the disease-free state:

$$
\frac{d I}{d t}=\beta(t)(1-r) I(t)-\gamma(t) I(t)
$$

We consider here the general case, not just the special case with $r=0$ and a constant $\gamma(t)$. Recall that the asymptotic growth rate of (4) is $\rho=\bar{\beta}(1-r)-\bar{\gamma}$ and is the unique real number such that the equation

$$
\frac{d J}{d t}+\rho J(t)=\beta(t)(1-r) J(t)-\gamma(t) J(t)
$$

has a nonzero periodic solution $J(t)$, as can be seen by setting $I(t)=J(t) \exp (\rho t)$ in $(4)$. $[13, \S 2]$ showed that the reproductive value in time-periodic linear population models such as (4) does not depend on "age" (here, infection age) and is given by any nonzero solution of the adjoint equation

$$
-\frac{d V}{d t_{0}}+\rho V\left(t_{0}\right)=\beta\left(t_{0}\right)(1-r) V\left(t_{0}\right)-\gamma\left(t_{0}\right) V\left(t_{0}\right) .
$$

This yields

$$
V\left(t_{0}\right)=\exp \left[\int_{0}^{t_{0}}(\gamma(t)-\bar{\gamma}) d t-(1-r) \int_{0}^{t_{0}}(\beta(t)-\bar{\beta}) d t\right]
$$


up to a multiplicative constant. Fig. 3 b compared to Fig. 3 a with $\mathcal{R}_{0}=1$ shows that the reproductive value gives only a crude idea of the dependence of the final epidemic size $R^{*}$ on $t_{0}$ : we can just expect that the maximum of $R^{*}$ is reached close to $t_{0}=0$ and the minimum close to $t_{0}=0.5$. With $\mathcal{R}_{0}=1.5$, the shape of $V\left(t_{0}\right)$ is similar with a maximum at $t_{0}=0$ and a minimum at $t_{0}=0.5$ (not shown) but Fig. 3a shows that this is misleading: the nonlinear effects become important. With a longer infectious period $(1 / \gamma=3$ weeks $)$, the difference between starting the epidemic at an unfavorable and at a favorable season is less pronounced than with a shorter infectious period $(1 / \gamma=1$ week).

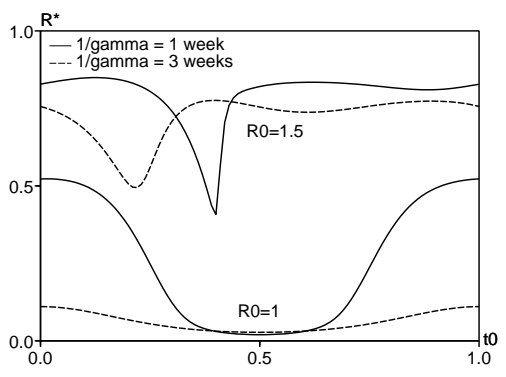

(a)

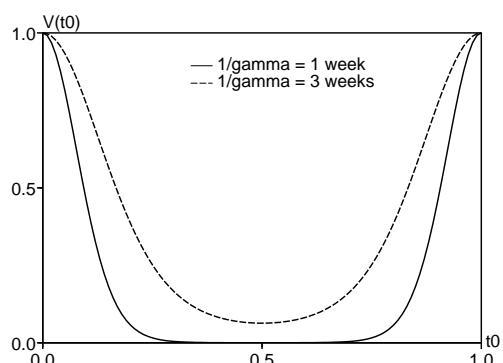

(b)

Figure 3: (a) When $\mathcal{R}_{0}$ is close to 1 , the final epidemic size $R^{*}$ depends strongly on $t_{0}$ if the infectious period $1 / \gamma$ is short compared to the seasonal period $\tau$. (b) The normalized "reproductive value" $V\left(t_{0}\right)$ gives a crude idea of the dependence of the final epidemic size on $t_{0}$ (here, $\mathcal{R}_{0}=1$ ).

Finally some remarks are necessary concerning a possible method estimation of $\mathcal{R}_{0}$ from data that does not use the final epidemic size. At the very beginning of an epidemic, $t \simeq t_{0}, S \simeq 1, I \simeq 0$ and $R \simeq 0$. So $d I / d t \simeq\left(\beta\left(t_{0}\right)-\gamma\right) I$ and $I(t)$ tends to grow exponentially at a rate $\beta\left(t_{0}\right)-\gamma$. This rate can be estimated from the beginning of the epidemic curve. Knowing the infectious period $1 / \gamma$, one can deduce $\beta\left(t_{0}\right)$ and therefore the ratio $\beta\left(t_{0}\right) / \gamma$. But our analysis shows that unlike $\mathcal{R}_{0}=\bar{\beta} / \gamma$, the ratio $\beta\left(t_{0}\right) / \gamma$ is not related to threshold properties of the system so it does not seem to be a good candidate for being called "basic reproduction number". If however $\beta(t)=\bar{\beta} f(t)$, where $f(t)$ is known and periodic with a mean equal to 1 , then $\mathcal{R}_{0}=\left(\beta\left(t_{0}\right) / \gamma\right) / f\left(t_{0}\right)$ can be computed. Notice that $\beta\left(t_{0}\right) / \gamma$ overestimates (resp. underestimates) $\mathcal{R}_{0}$ if $f\left(t_{0}\right)>1$ (resp. $f\left(t_{0}\right)<1$ ), i.e., if the epidemic starts during a favorable (resp. unfavorable) period where $\beta(t)$ is above (resp. below) its average $\bar{\beta}$. For air-borne diseases, it is difficult to know the shape of $f(t)=\beta(t) / \bar{\beta}$ because the influence of temperature and humidity on transmissibility is not easy to estimate quantitatively. For vectorborne diseases, the seasonal variations of the vector population can be measured so estimates of $\mathcal{R}_{0}$ can be obtained (see, e.g., [3]). 


\section{Threshold theorems}

\subsection{Periodic SIR system}

Prelimilary remarks. It follows from [2, $\S$ A.1] that (1)-(2) has a unique solution defined for all $t \geq t_{0}$ and that $S(t)>0$ and $I(t)>0$ for all $t \geq t_{0}$. Besides, $S(t)$ is decreasing, $R(t)$ is increasing and $S+I+R=1$. So $S(t) \rightarrow S^{*}$ and $R(t) \rightarrow R^{*}$ as $t \rightarrow+\infty$. Since $I=1-S-R$, it follows that $I(t) \rightarrow I^{*}$. But $R(t)-r=\int_{t_{0}}^{t} \gamma(u) I(u) d u$. So the latter integral converges as $t \rightarrow+\infty$ and $\bar{\gamma}>0$ implies that $I^{*}=0$.

Below the threshold. Assume that $\mathcal{R}_{0}<1$. Since $S(t)=1-I(t)-R(t)$, $I(t) \geq 0$ and $R(t) \geq r$ for all $t \geq t_{0}$, we have

$$
\frac{d I}{d t}=\beta(t)(1-I-R) I-\gamma(t) I \leq[\beta(t)(1-r)-\gamma(t)] I(t) .
$$

Since $I\left(t_{0}\right)=i$, we get

$$
I(t) \leq i \exp \left(\int_{t_{0}}^{t}[\beta(u)(1-r)-\gamma(u)] d u\right) .
$$

But $d R / d t=\gamma(t) I$ and $R\left(t_{0}\right)=r$. So

$$
r \leq R(t) \leq r+i \int_{t_{0}}^{t} \gamma(u) \exp \left(\int_{t_{0}}^{u}[\beta(v)(1-r)-\gamma(v)] d v\right) d u
$$

When $u \rightarrow+\infty$, we have $\int_{t_{0}}^{u}[\beta(v)(1-r)-\gamma(v)] d v \sim[\bar{\beta}(1-r)-\bar{\gamma}] u$. But $\bar{\beta}(1-r)-\bar{\gamma}<0$ since $\mathcal{R}_{0}<1$. So the integral on the right side of (5) converges as $t \rightarrow+\infty$ and

$$
r \leq R^{*} \leq r+i \int_{t_{0}}^{\infty} \gamma(u) \exp \left(\int_{t_{0}}^{u}[\beta(v)(1-r)-\gamma(v)] d v\right) d u
$$

So $R^{*}\left(t_{0}, i, r\right) \rightarrow r$ when $i \rightarrow 0$.

Above the threshold. Assume that $\mathcal{R}_{0}>1$. The proof goes on by contradiction. Suppose that $R^{*}-r<(1-r)\left(1-1 / \mathcal{R}_{0}\right)$. Then $1-R^{*}>(1-r) / \mathcal{R}_{0}=\bar{\gamma} / \bar{\beta}$. Since $R(t)$ is increasing, it follows that $R(t) \leq R^{*}$ for all $t \geq t_{0}$. Then

$$
\frac{d I}{d t}=\beta(t)(1-I-R) I-\gamma(t) I \geq \alpha(t) I-\beta(t) I^{2}
$$

where $\alpha(t)=\beta(t)\left(1-R^{*}\right)-\gamma(t)$. Moreover,

$$
\bar{\alpha}=\frac{1}{\tau} \int_{0}^{\tau} \alpha(t) d t=\bar{\beta}\left(1-R^{*}\right)-\bar{\gamma}>0 .
$$


Choose $\eta$ such that $0<\eta<\bar{\alpha} / \bar{\beta}$. Since $I(t) \rightarrow 0$ as $t \rightarrow+\infty$, one can find $t_{1}>t_{0}$ such that $0 \leq I(t) \leq \eta$ for all $t \geq t_{1}$. Now (6) implies that

$$
\frac{d I}{d t} \geq(\alpha(t)-\beta(t) \eta) I
$$

for all $t \geq t_{1}$. So $I(t) \geq I\left(t_{1}\right) \exp \left(\int_{t_{1}}^{t}(\alpha(u)-\beta(u) \eta) d u\right)$ for all $t \geq t_{1}$. Because of the choice of $\eta$, we obtain that $I(t) \rightarrow+\infty$ as $t \rightarrow+\infty$, which contradicts $I(t) \leq 1$. Therefore, $R^{*}-r \geq(1-r)\left(1-1 / \mathcal{R}_{0}\right)$.

\subsection{Periodic SEIR system}

Model and definition of $\mathcal{R}_{0}$. Consider the system

$\frac{d S}{d t}=-\beta(t) S I, \quad \frac{d E}{d t}=\beta(t) S I-\delta(t) E, \quad \frac{d I}{d t}=\delta(t) E-\gamma(t) I, \quad \frac{d R}{d t}=\gamma(t) I$,

with $S+E+I+R=1$ and where the rate $\delta(t)$ of moving from the latent compartment $E$ to the infectious compartment $I$ may also be $\tau$-periodic with $\bar{\delta}>0$. Consider the initial condition

$$
S\left(t_{0}\right)=1-e-i-r, \quad E\left(t_{0}\right)=e, \quad I\left(t_{0}\right)=i, \quad R\left(t_{0}\right)=r,
$$

with $e \geq 0, i \geq 0, r \geq 0, e+i>0$, and $e+i+r<1$. For any $\lambda>0$, let $\Phi\left(t, t_{0} ; \lambda\right)$ be the evolution operator associated with the $\tau$-periodic linear system

$$
\frac{d}{d t}\left(\begin{array}{c}
\tilde{E} \\
\tilde{I}
\end{array}\right)=\left(\begin{array}{cc}
-\delta(t) & \frac{\beta(t)(1-r)}{\lambda} \\
\delta(t) & -\gamma(t)
\end{array}\right)\left(\begin{array}{c}
\tilde{E} \\
\tilde{I}
\end{array}\right) .
$$

The spectral radius $\sigma(\lambda)$ of $\Phi\left(t_{0}+\tau, t_{0} ; \lambda\right)$ is called the dominant Floquet multiplier of (7) and does not depend on $t_{0}$. The off-diagonal rates of (7) being positive, [14, Lemma 2] implies that $\Phi\left(t, t_{0} ; \lambda\right)$ is a positive matrix for $t>t_{0}$. Moreover, $\sigma(\lambda)$ is a decreasing function of $\lambda[6]$. In [4, §3.4] (see also [6]), the basic reproduction number $\mathcal{R}_{0}$ was defined as the unique $\lambda>0$ such that $\sigma(\lambda)=1$.

Some remarks. It follows from $[2, \S$ A.1] that the periodic SEIR system has a unique solution defined for all $t \geq t_{0}$ and that $S(t)>0, E(t)>0$ and $I(t)>0$ for all $t>t_{0} . S(t)$ decreases and converges to $S^{*}$. $R(t)$ increases and converges to $R^{*}$. Since $\frac{d}{d t}(I+R)=\delta(t) E$, the function $I+R$ increases and converges. So $I(t) \rightarrow I^{*}$. Moreover, $R(t)-r=\int_{t_{0}}^{t} \gamma(u) I(u) d u$ converges as $t \rightarrow+\infty$. So $\bar{\gamma}>0$ implies that $I^{*}=0$. But $E=1-S-I-R$ shows that $E(t) \rightarrow E^{*}$. Since $\frac{d}{d t}(S+E)=-\delta(t) E$, the integral $\int_{t_{0}}^{\infty} \delta(u) E(u) d u$ converges. So $\bar{\delta}>0$ implies that $E^{*}=0$. Let us show that $S^{*}>0$. Imagine that $S^{*}=0$. Then

$$
\log S(t)-\log S\left(t_{0}\right)=-\int_{t_{0}}^{t} \beta(u) I(u) d u
$$


shows that $\int_{t_{0}}^{\infty} \beta(u) I(u) d u=+\infty$. But the inequalities

$$
\begin{aligned}
& \int_{t_{0}}^{t} \beta(u) I(u) d u \leq\left[\max _{0 \leq u \leq \tau} \frac{\beta(u)}{\gamma(u)}\right] \int_{t_{0}}^{t} \gamma(u) I(u) d u \\
& \int_{t_{0}}^{t} \gamma(u) I(u) d u=R(t)-r \leq 1-r
\end{aligned}
$$

show that $\int_{t_{0}}^{\infty} \beta(u) I(u) d u<+\infty$. Hence, $S^{*}>0$ and $R^{*}=1-S^{*}<1$.

Below the threshold. Since $S=1-E-I-R$, we have

$$
\frac{d}{d t}\left(\begin{array}{c}
E \\
I
\end{array}\right) \leq\left(\begin{array}{cc}
-\delta(t) & \beta(t)(1-r) \\
\delta(t) & -\gamma(t)
\end{array}\right)\left(\begin{array}{c}
E \\
I
\end{array}\right)
$$

where the inequality between vectors means inequality for each component. So $(E(t), I(t))^{\prime} \leq \Phi\left(t, t_{0} ; 1\right)(e, i)^{\prime}$, where the prime' stands for transposition. Assume that $\mathcal{R}_{0}<1$. Then $\sigma(1)<1$ and the matrix $\Phi\left(t, t_{0} ; 1\right)$ is bounded in norm by $K \exp \left(-\xi\left(t-t_{0}\right)\right)$ with $K>0$ and $\xi>0$ [15, Theor. 7.2]. So $R^{*}-r=\int_{0}^{\infty} \gamma(t) I(t) d t$ will tend to 0 if $e$ and $i$ tend to 0 .

Above the threshold. Assume $\mathcal{R}_{0}>1$. Imagine that the inequality $R^{*}-$ $r \geq(1-r)\left(1-1 / \mathcal{R}_{0}\right)$ is wrong. Then $1-R^{*}>(1-r) / \mathcal{R}_{0}$ and $\sigma((1-$ $\left.r) /\left(1-R^{*}\right)\right)>\sigma\left(R_{0}\right)=1$. By continuity of the spectral radius and because $R^{*}<1$, one can find $\eta>0$ such that $\eta<1-R^{*}$ and $\sigma(\lambda)>1$, where $\lambda=(1-r) /\left(1-R^{*}-\eta\right)$. We have $S(t) \rightarrow 1-R^{*}$ as $t \rightarrow+\infty$. So there is a $t_{1}>t_{0}$ such that $S(t) \geq 1-R^{*}-\eta$ for all $t \geq t_{1}$. It follows that

$$
\frac{d}{d t}\left(\begin{array}{c}
E \\
I
\end{array}\right) \geq\left(\begin{array}{cc}
-\delta(t) & \beta(t)\left(1-R^{*}-\eta\right) \\
\delta(t) & -\gamma(t)
\end{array}\right)\left(\begin{array}{c}
E \\
I
\end{array}\right)
$$

and $(E(t), I(t))^{\prime} \geq \Phi\left(t, t_{1} ; \lambda\right)\left(E\left(t_{1}\right), I\left(t_{1}\right)\right)^{\prime}$ for all $t \geq t_{1}$. In particular,

$$
\left(\begin{array}{c}
E\left(t_{1}+n \tau\right) \\
I\left(t_{1}+n \tau\right)
\end{array}\right) \geq \Phi\left(t_{1}+n \tau, t_{1} ; \lambda\right)\left(\begin{array}{c}
E\left(t_{1}\right) \\
I\left(t_{1}\right)
\end{array}\right)=\Phi\left(t_{1}+\tau, t_{1} ; \lambda\right)^{n}\left(\begin{array}{c}
E\left(t_{1}\right) \\
I\left(t_{1}\right)
\end{array}\right)
$$

for all integer $n \geq 1$. Let $\mu_{1}$ and $\mu_{2}$ be the eigenvalues of the positive matrix $\Phi\left(t_{1}+\tau, t_{1} ; \lambda\right)$, where $\mu_{1}=\sigma(\lambda)$ is the dominant eigenvalue of Perron-Frobenius theory [16]. Using Liouville's formula, we know that

$\operatorname{det}\left[\Phi\left(t_{1}+\tau, t_{1} ; \lambda\right)\right]=\mu_{1} \mu_{2}=\exp \left(-\int_{0}^{\tau}[\delta(t)+\gamma(t)] d t\right)=\exp (-(\bar{\delta}+\bar{\gamma}) \tau)<1$

Since $\mu_{1}=\sigma(\lambda)>1$, it follows that $\mu_{2}$ is real and $0<\mu_{2}<1$. Let $\left(p_{1,1}, p_{2,1}\right)^{\prime}$ be a positive eigenvector of the positive matrix $\Phi\left(t_{1}+\tau, t_{1} ; \lambda\right)$ associated with the eigenvalue $\mu_{1}$, as given by Perron-Frobenius theory. Let $\left(p_{1,2}, p_{2,2}\right)^{\prime}$ be a (real) eigenvector associated with $\mu_{2}$. As nonnegative eigenvectors can only be 
associated with $\mu_{1}\left[16\right.$, Theor. 2.1.4], it follows that $p_{1,2} p_{2,2}<0$. So one can assume that $p_{2,2}>0$ and $p_{1,2}<0$. Set

$$
P=\left(\begin{array}{ll}
p_{1,1} & p_{1,2} \\
p_{2,1} & p_{2,2}
\end{array}\right)
$$

Then $\Phi\left(t_{1}+\tau, t_{1} ; \lambda\right)^{n}=P \operatorname{diag}\left(\mu_{1}^{n}, \mu_{2}^{n}\right) P^{-1}$ for all integer $n \geq 1$. Let $\Delta=$ $p_{1,1} p_{2,2}-p_{1,2} p_{2,1}>0$ be the determinant of $P$. Then

$$
\begin{gathered}
\left(\begin{array}{c}
E\left(t_{1}+n \tau\right) \\
I\left(t_{1}+n \tau\right)
\end{array}\right) \geq \frac{1}{\Delta}\left(\begin{array}{ll}
p_{1,1} & p_{1,2} \\
p_{2,1} & p_{2,2}
\end{array}\right)\left(\begin{array}{cc}
\mu_{1}^{n} & 0 \\
0 & \mu_{2}^{n}
\end{array}\right)\left(\begin{array}{cc}
p_{2,2} & -p_{1,2} \\
-p_{2,1} & p_{1,1}
\end{array}\right)\left(\begin{array}{c}
E\left(t_{1}\right) \\
I\left(t_{1}\right)
\end{array}\right) \\
=\frac{1}{\Delta}\left(\begin{array}{l}
\mu_{1}^{n} p_{1,1}\left[p_{2,2} E\left(t_{1}\right)-p_{1,2} I\left(t_{1}\right)\right]+\mu_{2}^{n} p_{1,2}\left[-p_{2,1} E\left(t_{1}\right)+p_{1,1} I\left(t_{1}\right)\right] \\
\mu_{1}^{n} p_{2,1}\left[p_{2,2} E\left(t_{1}\right)-p_{1,2} I\left(t_{1}\right)\right]+\mu_{2}^{n} p_{2,2}\left[-p_{2,1} E\left(t_{1}\right)+p_{1,1} I\left(t_{1}\right)\right]
\end{array}\right) .
\end{gathered}
$$

It follows from $\mu_{1}>1,0<\mu_{2}<1, \Delta>0, p_{1,1}>0, p_{2,1}>0$ and $p_{2,2} E\left(t_{1}\right)-$ $p_{1,2} I\left(t_{1}\right)>0$ that both $E\left(t_{1}+n \tau\right)$ and $I\left(t_{1}+n \tau\right)$ tend to $+\infty$ as $n \rightarrow+\infty$. But this contradicts $(E(t), I(t)) \rightarrow(0,0)$ as $t \rightarrow+\infty$. So $R^{*}-r \geq(1-r)\left(1-1 / \mathcal{R}_{0}\right)$.

\subsection{A periodic system describing vector-borne diseases}

Consider the system describing a vector-borne disease

$$
\frac{d S}{d t}=-\frac{\beta S J}{H}, \quad \frac{d I}{d t}=\frac{\beta S J}{H}-\gamma I, \quad \frac{d R}{d t}=\gamma I, \quad \frac{d J}{d t}=\beta^{\prime}(V(t)-J) I-\delta J,
$$

with a periodic vector population $V(t)$, and where $H$ is the total human population, $S+I+R=1, J$ is the number (not the fraction) of infected vectors, $\delta$ is the vector mortality, and $\beta$ (resp. $\beta^{\prime}$ ) is the vector biting rate multiplied by the transmission probability from vector to human (resp. from human to vector). This is reasonable model for arbovirus epidemics: dengue fever, West Nile fever, yellow fever, chikungunya.. The initial condition is $S\left(t_{0}\right)=1-i-r, I\left(t_{0}\right)=i$, $R\left(t_{0}\right)=r, J\left(t_{0}\right)=j$, with $i>0, r \geq 0, i+r<1$, and $0 \leq j \leq V\left(t_{0}\right)$. The basic reproduction number $\mathcal{R}_{0}$ is such that system

$$
\frac{d}{d t}\left(\begin{array}{c}
\tilde{I} \\
\tilde{J}
\end{array}\right)=\left(\begin{array}{cc}
-\gamma & \frac{\beta(1-r)}{\mathcal{R}_{0} H} \\
\beta^{\prime} V(t) & -\delta
\end{array}\right)\left(\begin{array}{c}
\tilde{I} \\
\tilde{J}
\end{array}\right)
$$

has a dominant Floquet multiplier equal to 1 [4] (some authors prefer to use $\left.\mathcal{R}_{0}^{\prime}=\sqrt{\mathcal{R}_{0}}\right)$. One can show as in $\S 3.2$ that the final size $R^{*}-r$ of the epidemic in humans tends to 0 when $\mathcal{R}_{0}<1$ and both $i$ and $j$ tend to 0 , and that $R^{*}-r \geq(1-r)\left(1-1 / \mathcal{R}_{0}\right)$ if $\mathcal{R}_{0}>1$. We briefly sketch the proof. When $\mathcal{R}_{0}<1$, the result follows from the fact that

$$
\frac{d}{d t}\left(\begin{array}{l}
I \\
J
\end{array}\right) \leq\left(\begin{array}{cc}
-\gamma & \frac{\beta(1-r)}{H} \\
\beta^{\prime} V(t) & -\delta
\end{array}\right)\left(\begin{array}{l}
I \\
J
\end{array}\right) .
$$

When $\mathcal{R}_{0}>1$, we have $R(t) \rightarrow R^{*}, S(t) \rightarrow 1-R^{*}, I(t) \rightarrow 0$, and $J(t) \rightarrow 0$ as $t \rightarrow+\infty$. Assume that $1-R^{*}>(1-r) / \mathcal{R}_{0}$. Then one can find $\eta>0$ and 
$t_{1}>t_{0}$ such that

$$
\frac{d}{d t}\left(\begin{array}{l}
I \\
J
\end{array}\right) \geq\left(\begin{array}{cc}
-\gamma & \frac{\beta\left(1-R^{*}-\eta\right)}{H} \\
\beta^{\prime}(V(t)-\eta) & -\delta
\end{array}\right)\left(\begin{array}{l}
I \\
J
\end{array}\right) .
$$

for all $t \geq t_{1}$, the dominant Floquet multiplier of the right side being strictly bigger than 1. This leads as in $\S 3.2$ to a contradiction with $I(t) \leq 1$. So $1-R^{*} \leq(1-r) / \mathcal{R}_{0}$.

\section{Conclusion}

Our analysis shows that the threshold theorem for systems with constant coefficients (with the two classical cases, $\mathcal{R}_{0}<1$ and $\mathcal{R}_{0}>1$ ) can be generalized to systems with periodic coefficients representing seasonality, provided the basic reproduction number $\mathcal{R}_{0}$ is defined as in our earlier studies $[3,4,5]$. However, somewhat unexpectedly, periodic systems may have relatively large epidemics even when $\mathcal{R}_{0}<1$ and the final epidemic size may not increase with $\mathcal{R}_{0}$ or the initial fraction $i$ of infected people.

These observations based on simple systems should serve as a warning for the interpretation of epidemics influenced by seasonality. Epidemics of emerging vector-borne diseases, which receive a renewed attention because of climate change theory, should be analyzed with caution as we have shown with the case of chikungunya in Réunion and Italy. Another case of interest nowadays is that of pandemic flu in humans following bird flu. The pandemic of 1918-1919 occured in several waves influenced by seasonality. Attempts to estimate the basic reproduction number for this pandemic have assumed constant coefficients and have used the beginning of the epidemic curve or the final size of singlewave epidemics (see, e.g., [17]). Our work suggests that these analyses may have to be revised since the relationship between $\mathcal{R}_{0}$ and the behavior of epidemics influenced by seasonality is not a straightforward generalization of what is known for the case of a constant environment.

\section{Acknowledgment}

This work was initiated while N.B. was visiting Instituto Gulbenkian de Ciência, with funding from the Portuguese Research Council (FCT) and the European Commission (grant MEXT-CT-2004-14338). N.B. also thanks the European CDC for its invitation to a chikungunya modeling meeting.

\section{References}

[1] W. O. Kermack, A. G. McKendrick, (1927), A contribution to the mathematical theory of epidemics, Proc. Roy. Soc. 115A, 700-721. 
[2] H. R. Thieme (2003) Mathematics in Population Biology, Princeton University Press, Princeton.

[3] N. Bacaër, S. Guernaoui, (2006), The epidemic threshold of vector-borne diseases with seasonality, J. Math. Biol. 53, 421-436.

[4] N. Bacaër, (2007), Approximation of the basic reproduction number $R_{0}$ for vector-borne diseases with a periodic vector population, Bull. Math. Biol. 69, 1067-1091.

[5] N. Bacaër, R. Ouifki, (2007), Growth rate and basic reproduction number for population models with a simple periodic factor, Math. Biosci. 210, $647-658$.

[6] W. Wang, X.-Q. Zhao, (2008), Threshold dynamics for compartmental epidemic models in periodic environments, J. Dyn. Diff. Equat. 20, 699-717.

[7] N. Bacaër, Periodic matrix population models: growth rate, basic reproduction number and entropy. Submitted.

[8] O. Diekmann, J. A. P. Heesterbeek (2000) Mathematical Epidemiology of Infectious Diseases, Wiley, Chichester.

[9] European Centre for Disease Prevention and Control (2009) Report, Expert Meeting on Chikungunya Modelling, ECDC, Stockholm.

[10] R. M. Anderson, R. M. May (1991) Infectious Diseases of Humans: Dynamics and Control, Oxford University Press, Oxford.

[11] J. Ma, D. J. D. Earn, (2006), Generality of the final size formula for an epidemic of a newly invading infectious disease, Bull. Math. Biol. 68, 679702 .

[12] J. Arino, F. Brauer, P. van den Driessche, J. Watmough, J. Wu, (2007), A final size relation for epidemic models, Math. Biosci. Eng. 4, 159-175.

[13] N. Bacaër, X. Abdurahman, (2008), Resonance of the epidemic threshold in a periodic environment, J. Math. Biol. 57, 649-673.

[14] G. Aronsson, R. B. Kellogg, (1978), On a differential equation arising from compartmental analysis, Math. Biosci. 38, 113-122.

[15] J. K. Hale (1980) Ordinary Differential Equations, Krieger, Malabar.

[16] A. Berman, R. J. Plemmons, (1979), Nonnegative Matrices in the Mathematical Sciences, Academic Press, New York.

[17] E. Vynnycky, A. Trindall, P. Mangtani, (2007), Estimates of the reproduction numbers of Spanish influenza using morbidity data, Int. J. Epidem. $36,881-889$. 


\title{
On the final size of epidemics with seasonality
}

\author{
Nicolas BACAËR* M. Gabriela M. GOMES ${ }^{\ddagger}$
}

\begin{abstract}
We first study an SIR system of differential equations with periodic coefficients describing an epidemic in a seasonal environment. Unlike in a constant environment, the final epidemic size may not be an increasing function of the basic reproduction number $\mathcal{R}_{0}$ or of the initial fraction of infected people. Moreover, large epidemics can happen even if $\mathcal{R}_{0}<1$. But like in a constant environment, the final epidemic size tends to 0 when $\mathcal{R}_{0}<1$ and the initial fraction of infected people tends to 0 . When $\mathcal{R}_{0}>1$, the final epidemic size is bigger than the fraction $1-1 / \mathcal{R}_{0}$ of the initially nonimmune population. In summary, the basic reproduction number $\mathcal{R}_{0}$ keeps its classical threshold property but many other properties are no longer true in a seasonal environment. These theoretical results should be kept in mind when analyzing data for emerging vector-borne diseases (West-Nile, dengue, chikungunya) or air-borne diseases (SARS, pandemic influenza), all these diseases being influenced by seasonality.
\end{abstract}

Keywords: basic reproduction number, seasonality, final epidemic size.

\section{Introduction}

Consider the following SIR system describing an epidemic

$$
\frac{d S}{d t}=-\beta(t) S I, \quad \frac{d I}{d t}=\beta(t) S I-\gamma(t) I, \quad \frac{d R}{d t}=\gamma(t) I,
$$

with continuous positive $\tau$-periodic contact rate $\beta(t)$ and recovery rate $\gamma(t)$. The function $S(t)$ is the fraction of the population that is susceptible (i.e., not yet infected), $I(t)$ the fraction that is infected, $R(t)$ the fraction that has recovered from infection and become immune, so that $S(t)+I(t)+R(t)=1$. Consider the initial condition

$$
S\left(t_{0}\right)=1-i-r, \quad I\left(t_{0}\right)=i, \quad R\left(t_{0}\right)=r
$$

\footnotetext{
* Corresponding author. IRD (Institut de Recherche pour le Développement), 32 avenue Henri Varagnat, 93413 Bondy, France. E-mail: bacaer@bondy.ird.fr

†Instituto Gulbenkian de Ciência, Apartado 14, 2781-901 Oeiras, Portugal.

${ }^{\ddagger}$ Centro de Matemática e Aplicações Fundamentais, Universidade de Lisboa, Av. Prof. Gama Pinto 2, 1649-003 Lisboa, Portugal.
} 
with $i>0, r \geq 0$ and $i+r<1$. Notice that the trivial cases $i=0$ and $i+r=1$ are excluded and that the special case $r=0$ corresponds to emerging diseases for which the population has no immunity. Let $R^{*}$ be the limit of $R(t)$ as $t \rightarrow+\infty$. Then $R^{*}-r$ is the final epidemic size. $R^{*}$ depends on the functions $\beta(t)$ and $\gamma(t)$ and on the parameters $t_{0}, i$ and $r$. To emphasize this dependence, we could write $R^{*}=R^{*}\left(\beta(\cdot), \gamma(\cdot), t_{0}, i, r\right)$. System (1) with a periodic $\beta(t)$ and a constant $\gamma$ can be used for epidemics of viral air-borne diseases that occur on a fast time scale compared to demographic processes and to the immune period, such as influenza (flu) and SARS.

When $\beta(t)$ and $\gamma(t)$ are both constant, (1) is the "simplified KermackMcKendrick system" $[1,2]$. In this special case, there is a closed but implicit formula for $R^{*}$ :

$$
\left(1-R^{*}\right) \exp \left[\mathcal{R}_{0} \frac{R^{*}-r}{1-r}\right]=1-i-r,
$$

where $\mathcal{R}_{0}=\beta / \gamma$ is the "basic reproduction number". It follows that $R^{*}$ is an increasing function of $\mathcal{R}_{0}$, that it is independent of $t_{0}$, and that it is an increasing function of $i$. All these properties are quite intuitive. If $\mathcal{R}_{0}<1$ then $R^{*} \rightarrow r$ when $i \rightarrow 0$. If $\mathcal{R}_{0}>1$ then

$$
R^{*}-r \geq(1-r)\left(1-1 / \mathcal{R}_{0}\right),
$$

as is easily checked by studying the left side of (3) as a function of $R^{*}$ (see also [2, Theor. 18.6]). $R^{*}$ converges when $i \rightarrow 0$ to a positive limit if $\mathcal{R}_{0}>1$. Assuming $r=0$ (emerging disease), this limit can be identified with the result of a post-epidemic seroprevalence survey. Then (3) gives an estimate of $\mathcal{R}_{0}$, which in turn can give an estimate of the vaccination coverage necessary to prevent an epidemic of the same disease in other areas with similar characteristics.

The problem of the definition of the basic reproduction number for periodic systems has been investigated in $[3,4,5,6,7]$. In summary, we have for system (1)

$$
\mathcal{R}_{0}=\frac{\bar{\beta}(1-r)}{\bar{\gamma}}, \quad \bar{\beta}=\frac{1}{\tau} \int_{0}^{\tau} \beta(t) d t, \quad \bar{\gamma}=\frac{1}{\tau} \int_{0}^{\tau} \gamma(t) d t .
$$

Indeed, linearizing (1) near the disease-free steady state $(S=1-r, I=$ $0, R=r)$, we see that $d I / d t \simeq \beta(t)(1-r) I-\gamma(t) I$. $\mathcal{R}_{0}=1$ is obviously a threshold for this simple linear periodic equation. But one can also show that $\mathcal{R}_{0}$ is the spectral radius of the next-generation integral operator acting on periodic continuous functions

$$
\phi(t) \longmapsto \int_{0}^{\infty} K(t, x) \phi(t-x) d x,
$$

where $K(t, x)=\beta(t)(1-r) \exp \left(-\int_{t-x}^{t} \gamma(s) d s\right)$ is the rate of secondary cases produced at time $t$ by somebody infected at time $t-x[3, \S 5]$. This approach is close to the "usual" definition of $\mathcal{R}_{0}$ in a constant environment as the average number of secondary cases produced by 
an initial case. But seasonality introduces a level of complexity similar to that in age-structured epidemic models, for which $\mathcal{R}_{0}$ is again the spectral radius of an integral operator [8]. It is also easily shown that $\mathcal{R}_{0}$ is the unique real positive number such that the linear periodic system $d I / d t=\beta(t)(1-r) I / \mathcal{R}_{0}-\gamma(t) I$ has a dominant Floquet multiplier equal to 1 (see $[4, \S 3.4]$ and $[6]$ ). $\mathcal{R}_{0}$ appears also in the analysis of periodic birth and death processes $[4, \S 5.2]$. Notice that we call $\mathcal{R}_{0}$ the basic reproduction number, while some authors would call it the effective reproduction number and keep $\mathcal{R}_{0}$ for the ratio $\bar{\beta} / \bar{\gamma}$. In any case, $\mathcal{R}_{0}$ does not depend on $i$ and $t_{0}$.

In Section 2 we start investigating which properties of the simplified KermackMcKendrick system remain true in the periodic variant (1). It turns out that $R^{*}$ may not be an increasing function of $\mathcal{R}_{0}$, that it is a $\tau$-periodic function of $t_{0}$, and that it may not be an increasing function of $i$. The first and third properties are somewhat counterintuitive. The first property implies that it may be impossible to estimate $\mathcal{R}_{0}$ from seroprevalence data. Simulations also show that large epidemics can occur even when $\mathcal{R}_{0}<1$. This happens if the disease is introduced during a favorable period, if the initial fraction of infected people is not too small, if seasonality is sufficiently marked, and if the average infectious period $1 / \gamma$ is short compared to the length $\tau$ of the season. The 2007 chikungunya epidemic in Italy may have been one such case [9]. One should not conclude that $\mathcal{R}_{0}>1$ just because an epidemic peak is observed and one should be careful at how $\mathcal{R}_{0}$ is defined if seasonality is believed to be important. Simulations also show that the final epidemic size can be very sensitive to small changes in $\mathcal{R}_{0}$. This may explain why it is so difficult to predict the future of epidemics influenced by seasonality, as noticed during the 2005-2006 chikungunya epidemic in Réunion (an island of the Indian ocean).

In section 3 we show that, as in the simplified Kermack-McKendrick system, $\mathcal{R}_{0}=1$ is a threshold for the nonlinear periodic system (1). More precisely, we show that

- if $\mathcal{R}_{0}<1$, then $R^{*}-r \rightarrow 0$ when $i \rightarrow 0$.

- if $\mathcal{R}_{0}>1$, then $R^{*}-r \geq(1-r)\left(1-1 / \mathcal{R}_{0}\right)$ for all $0<i<1-r$.

Notice that for the case $\mathcal{R}_{0}>1$, we have $1-R^{*} \leq(1-r) / \mathcal{R}_{0}$. So the epidemic divides the initial nonimmune population by a number greater than $\mathcal{R}_{0}$. In some sense, this is like classical vaccination theory for systems with constant coefficients [10]. Similar threshold theorems have been or can be derived for various generalizations of the simplified Kermack-McKendrick system [1, 2, 8, $10,11,12]$. But our method of proof will be different because we could not find any final-size equation similar to (3) for systems with periodic coefficients. In section 3 we also prove that the threshold theorem remains true for a periodic SEIR system and for a periodic system describing vector-borne diseases, $\mathcal{R}_{0}$ being most easily defined and computed as in $[4, \S 3.4]$ (see also [6]). 


\section{Numerical simulations}

To keep things simple and because of the widespread interest in pandemic flu, we will use the periodic SIR system although the discussion will be extended to a vector-borne disease (chikungunya). One can check that similar qualitative remarks can be made using the system of $\S 3.3$. So let us consider (1) with for example $\beta(t)=\bar{\beta}(1+\varepsilon \sin 2 \pi t / \tau)$, where $\tau=1$ year represents seasonality and obviously cannot be changed. In this section, we shall assume that $r=0$ (emerging disease) and will investigate how $R^{*}$ depends on the other parameters: $\bar{\beta}, \varepsilon, \gamma, t_{0}$, and $i$.

Fig. 1a shows that the final epidemic size $R^{*}$ may not increase with the basic reproduction number $\mathcal{R}_{0}=\bar{\beta} / \gamma$. The parameter values are $\varepsilon=0.5$, $1 / \gamma=1$ week $=1 / 52$ year, $t_{0} / \tau=0.5, i=10^{-3}$, and we took two values for $\bar{\beta}$ corresponding to $\mathcal{R}_{0}=2$ and $\mathcal{R}_{0}=2.5$. With the higher value of $\mathcal{R}_{0}$, the epidemic occurs during the unfavorable season $0.5<t / \tau<1$, when $\beta(t)$ is below its average. When the favorable season arrives $(1<t / \tau<1.5)$, the stock of susceptibles has already been largely depleted so no new epidemic peak occurs. For the smaller value of $\mathcal{R}_{0}$, the stock of susceptibles has not been depleted enough, a second epidemic wave occurs and the final epidemic size is larger. The latter situation is precisely what happened in 2005-2006 in Réunion, a small island of the Indian ocean and a French overseas territory. A first small peak occured in May 2005, just before the beginning of "winter" in the southern hemisphere. The epidemic crossed the winter at a low level. A second big epidemic peak occured at the beginning of the next "summer" in January 2006 and infected about 250,000 people, one third of the island's population. Notice finally that if the final epidemic size $R^{*}$ is not a monotone increasing function of $\mathcal{R}_{0}$, then it is impossible to estimate $\mathcal{R}_{0}$ from $R^{*}$ and in particular from seroprevalence data. However, we will show in Section 3 that $R^{*}-r \geq$ $(1-r)\left(1-1 / \mathcal{R}_{0}\right)$. So we know at least that $\mathcal{R}_{0} \leq(1-r) /\left(1-R^{*}\right)$, which gives an upper bound for $\mathcal{R}_{0}$.

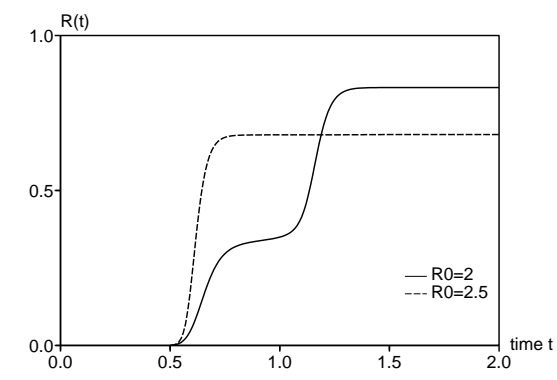

(a)

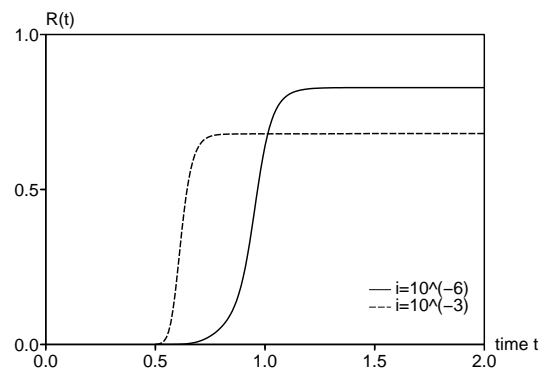

(b)

Figure 1: The final epidemic size may not increase with the basic reproduction number $\mathcal{R}_{0}$ (a) or with the initial fraction $i$ of infected people (b). 
Similarly, Fig. 1b shows that the final epidemic size $R^{*}$ may not increase with the initial fraction $i$ of infected people. The parameter values are $\varepsilon=0.5$, $1 / \gamma=1 / 52$ year, $t_{0} / \tau=0.5, \mathcal{R}_{0}=2.5$ (which fixes $\bar{\beta}$ ), and we took either $i=10^{-6}$ or $i=10^{-3}$. Again, $i=10^{-6}$ depletes the number of susceptibles more slowly during the unfavorable season.

Fig. 2a shows that large epidemics are possible even if $\mathcal{R}_{0}<1$. The parameter values are $\mathcal{R}_{0}=0.9, \varepsilon=0.5,1 / \gamma=1 / 52$ year, $t_{0} / \tau=0$, and $i=10^{-3}$. The fact that $\mathcal{R}_{0}(1+\varepsilon)>1$ but $\mathcal{R}_{0}(1-\varepsilon)<1$ gives an indication of what is happening [more generally, (1) shows that $d I / d t<0$ when $\beta(t) / \gamma(t)<1$ ]. The epidemic occurs during the favorable season and simply stops when the unfavorable period arrives. The fact that the initial fraction of infected people is not too small $\left(i=10^{-3}\right)$ also plays a role. Indeed, the threshold theorem with $r=0$ shows that $R^{*} \rightarrow 0$ when $i \rightarrow 0$ and $\mathcal{R}_{0}<1$. From these remarks, one concludes that one should be careful before saying that $\mathcal{R}_{0}>1$ whenever an epidemic peak is observed. In the summer of 2007, a small chikungunya epidemic occured near Ravenna in Italy. Summer is the best season for mosquitoes in that area and the epidemic could probably never have crossed the winter. In our opinion, the estimates of $\mathcal{R}_{0}$ presented during the chikungunya modeling meeting at the European Centre for Disease Control and Prevention, which were all well above 1 , should be considered with caution [9]. The problem lies essentially in the definition of $\mathcal{R}_{0}$ and in the assumptions of the model. A model with a constant environment similar to the summer conditions cannot explain why the epidemic does not continue during falls, and is clearly inappropriate if the epidemic lasts two years as in Réunion.

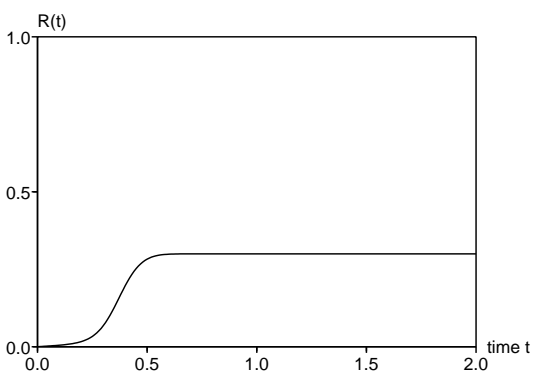

(a)

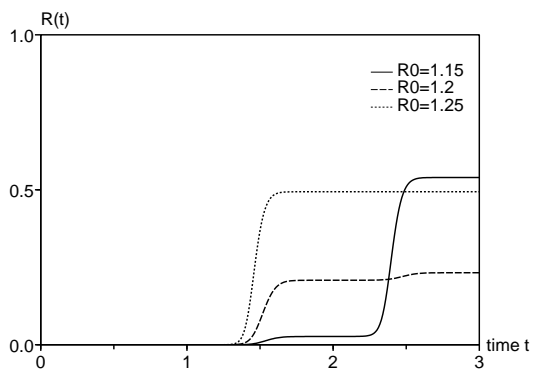

(b)

Figure 2: (a) Large epidemics can happen even if $\mathcal{R}_{0}<1$. (b) $R^{*}$ can be very sensitive to small changes in $\mathcal{R}_{0}$.

Fig. $2 \mathrm{~b}$ shows that the final epidemic size $R^{*}$ can be very sensitive to small changes in $\mathcal{R}_{0}$. The parameters values are $\varepsilon=0.5,1 / \gamma=1 / 52$ year, $t_{0} / \tau=$ $0.5, i=10^{-6}$, while $\mathcal{R}_{0}$ can take one of the three values 1.15 (plain line), 1.2 (dashed line), and 1.25 (dotted line). We obtained $R^{*} \simeq 54 \%$ when $\mathcal{R}_{0}=1.15$, $R^{*} \simeq 23 \%$ when $\mathcal{R}_{0}=1.2$, and $R^{*} \simeq 50 \%$ when $\mathcal{R}_{0}=1.25$. In practice, one may never be able to distinguish values of $\mathcal{R}_{0}$, which are so close. But the 
corresponding final epidemic size varies by a factor 2 . In systems with periodic coefficients such as (1), forecasting the final epidemic size seems very difficult. This can answer in some way the critics directed against the epidemiologists, who followed the chikungunya epidemic in Réunion. Although the epidemic had been carefully monitored by a surveillance network since its beginning in April 2005, epidemiologists were not able to foresee the huge peak that occured in January and February 2006. This led to much pressure set by the general public and politicians on the "Institut de Veille Sanitaire", which is in charge of disease surveillance in France and its overseas territories. Our simulations suggest that this pressure may have been unjustified. In some way, epidemic forecasting beyond a few weeks in a seasonal environment may be almost as difficult as weather forecasting beyond a few days. Recall that the difficulty of analyzing endemic (not emerging) diseases in a seasonal environment, chaos often being suspected, is a somewhat different question as the one studied here.

For Fig. 2b, we chose $i=10^{-6}$. In practice, it is difficult to estimate the initial fraction $i$ of infected people. The problem is that the SIR system assumes some kind of homogeneous mixing. If an epidemic starts in a city from just one initial case, one may think that $i$ is simply one over the population of the city. But if the city is large, homogeneous mixing may not be reasonable and we may have to use the population of the quarter of the city where the initial case was introduced. The problem is the same for epidemics in a small island like Réunion but with a population of 800,000 , which is concentrated along the coast.

Fig. 3a investigates the dependence of the final epidemic size $R^{*}$ on the time $t_{0}$ at which the epidemic starts. Obviously, $R^{*}$ is always a $\tau$-periodic function of $t_{0}$, system (1) being invariant by a shift of $\tau$ in time. The parameter values in Fig. 3 a are $\mathcal{R}_{0}=1$ or $\mathcal{R}_{0}=1.5$, $\varepsilon=0.5,1 / \gamma=1$ week or 3 weeks, and $i=10^{-3}$. The dependence on $t_{0}$ is strong if $\mathcal{R}_{0}$ is close to 1 and if the infectious period $1 / \gamma$ is short compared to the seasonal period $\tau$. In such a case, the epidemic cannot develop during the unfavorable season. Fig. 3b shows for $\mathcal{R}_{0}=1$ the "reproductive value" $V\left(t_{0}\right)$ ("infectious value" might be a more appropriate expression) of an initial case introduced at time $t_{0}$, computed from the linearized equation near the disease-free state:

$$
\frac{d I}{d t}=\beta(t)(1-r) I(t)-\gamma(t) I(t)
$$

We consider here the general case, not just the special case with $r=0$ and a constant $\gamma(t)$. Recall that the asymptotic growth rate of (4) is $\rho=\bar{\beta}(1-r)-\bar{\gamma}$ and is the unique real number such that the equation

$$
\frac{d J}{d t}+\rho J(t)=\beta(t)(1-r) J(t)-\gamma(t) J(t)
$$

has a nonzero periodic solution $J(t)$, as can be seen by setting $I(t)=$ $J(t) \exp (\rho t)$ in (4). [13, §2] showed that the reproductive value in time-periodic linear population models such as (4) does not depend 
on "age" (here, infection age) and is given by any nonzero solution of the adjoint equation

$$
-\frac{d V}{d t_{0}}+\rho V\left(t_{0}\right)=\beta\left(t_{0}\right)(1-r) V\left(t_{0}\right)-\gamma\left(t_{0}\right) V\left(t_{0}\right) .
$$

This yields

$$
V\left(t_{0}\right)=\exp \left[\int_{0}^{t_{0}}(\gamma(t)-\bar{\gamma}) d t-(1-r) \int_{0}^{t_{0}}(\beta(t)-\bar{\beta}) d t\right]
$$

up to a multiplicative constant. Fig. 3b compared to Fig. 3a with $\mathcal{R}_{0}=1$ shows that the reproductive value gives only a crude idea of the dependence of the final epidemic size $R^{*}$ on $t_{0}$ : we can just expect that the maximum of $R^{*}$ is reached close to $t_{0}=0$ and the minimum close to $t_{0}=0.5$. With $\mathcal{R}_{0}=1.5$, the shape of $V\left(t_{0}\right)$ is similar with a maximum at $t_{0}=0$ and a minimum at $t_{0}=0.5$ (not shown) but Fig. 3a shows that this is misleading: the nonlinear effects become important. With a longer infectious period $(1 / \gamma=3$ weeks $)$, the difference between starting the epidemic at an unfavorable and at a favorable season is less pronounced than with a shorter infectious period $(1 / \gamma=1$ week $)$.

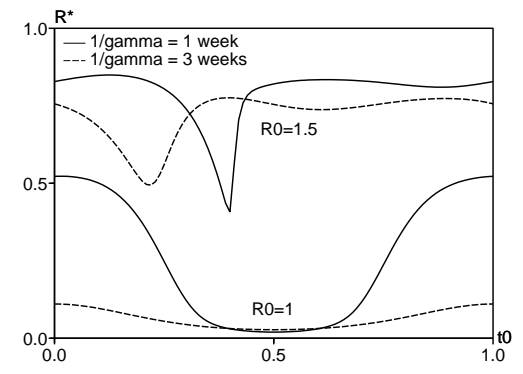

(a)

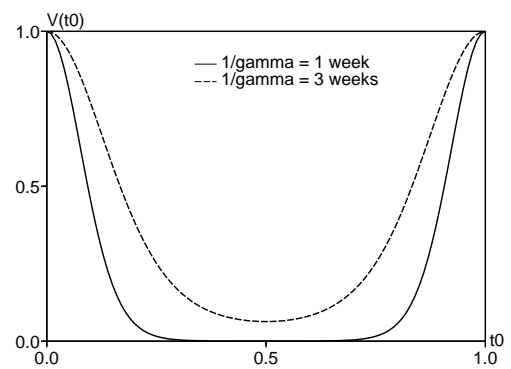

(b)

Figure 3: (a) When $\mathcal{R}_{0}$ is close to 1 , the final epidemic size $R^{*}$ depends strongly on $t_{0}$ if the infectious period $1 / \gamma$ is short compared to the seasonal period $\tau$. (b) The normalized "reproductive value" $V\left(t_{0}\right)$ gives a crude idea of the dependence of the final epidemic size on $t_{0}$ (here, $\mathcal{R}_{0}=1$ ).

Finally some remarks are necessary concerning a possible method estimation of $\mathcal{R}_{0}$ from data that does not use the final epidemic size. At the very beginning of an epidemic, $t \simeq t_{0}, S \simeq 1, I \simeq 0$ and $R \simeq 0$. So $d I / d t \simeq\left(\beta\left(t_{0}\right)-\gamma\right) I$ and $I(t)$ tends to grow exponentially at a rate $\beta\left(t_{0}\right)-\gamma$. This rate can be estimated from the beginning of the epidemic curve. Knowing the infectious period $1 / \gamma$, one can deduce $\beta\left(t_{0}\right)$ and therefore the ratio $\beta\left(t_{0}\right) / \gamma$. But our analysis shows that unlike $\mathcal{R}_{0}=\bar{\beta} / \gamma$, the ratio $\beta\left(t_{0}\right) / \gamma$ is not related to threshold properties of the system 
so it does not seem to be a good candidate for being called "basic reproduction number". If however $\beta(t)=\bar{\beta} f(t)$, where $f(t)$ is known and periodic with a mean equal to 1 , then $\mathcal{R}_{0}=\left(\beta\left(t_{0}\right) / \gamma\right) / f\left(t_{0}\right)$ can be computed. Notice that $\beta\left(t_{0}\right) / \gamma$ overestimates (resp. underestimates) $\mathcal{R}_{0}$ if $f\left(t_{0}\right)>1$ (resp. $f\left(t_{0}\right)<1$ ), i.e., if the epidemic starts during a favorable (resp. unfavorable) period where $\beta(t)$ is above (resp. below) its average $\bar{\beta}$. For air-borne diseases, it is difficult to know the shape of $f(t)=\beta(t) / \bar{\beta}$ because the influence of temperature and humidity on transmissibility is not easy to estimate quantitatively. For vectorborne diseases, the seasonal variations of the vector population can be measured so estimates of $\mathcal{R}_{0}$ can be obtained (see, e.g., [3]).

\section{Threshold theorems}

\subsection{Periodic SIR system}

Prelimilary remarks. It follows from [2, §A.1] that (1)-(2) has a unique solution defined for all $t \geq t_{0}$ and that $S(t)>0$ and $I(t)>0$ for all $t \geq t_{0}$. Besides, $S(t)$ is decreasing, $R(t)$ is increasing and $S+I+R=1$. So $S(t) \rightarrow S^{*}$ and $R(t) \rightarrow R^{*}$ as $t \rightarrow+\infty$. Since $I=1-S-R$, it follows that $I(t) \rightarrow I^{*}$.

But $R(t)-r=\int_{t_{0}}^{t} \gamma(u) I(u) d u$. So the latter integral converges as $t \rightarrow+\infty$ and $\bar{\gamma}>0$ implies that $I^{*}=0$.

Below the threshold. Assume that $\mathcal{R}_{0}<1$. Since $S(t)=1-I(t)-R(t)$, $I(t) \geq 0$ and $R(t) \geq r$ for all $t \geq t_{0}$, we have

$$
\frac{d I}{d t}=\beta(t)(1-I-R) I-\gamma(t) I \leq[\beta(t)(1-r)-\gamma(t)] I(t) .
$$

Since $I\left(t_{0}\right)=i$, we get

$$
I(t) \leq i \exp \left(\int_{t_{0}}^{t}[\beta(u)(1-r)-\gamma(u)] d u\right) .
$$

But $d R / d t=\gamma(t) I$ and $R\left(t_{0}\right)=r$. So

$$
r \leq R(t) \leq r+i \int_{t_{0}}^{t} \gamma(u) \exp \left(\int_{t_{0}}^{u}[\beta(v)(1-r)-\gamma(v)] d v\right) d u
$$

When $u \rightarrow+\infty$, we have $\int_{t_{0}}^{u}[\beta(v)(1-r)-\gamma(v)] d v \sim[\bar{\beta}(1-r)-\bar{\gamma}] u$. But $\bar{\beta}(1-r)-\bar{\gamma}<0$ since $\mathcal{R}_{0}<1$. So the integral on the right side of (5) converges as $t \rightarrow+\infty$ and

$$
r \leq R^{*} \leq r+i \int_{t_{0}}^{\infty} \gamma(u) \exp \left(\int_{t_{0}}^{u}[\beta(v)(1-r)-\gamma(v)] d v\right) d u .
$$

So $R^{*}\left(t_{0}, i, r\right) \rightarrow r$ when $i \rightarrow 0$. 
Above the threshold. Assume that $\mathcal{R}_{0}>1$. The proof goes on by contradiction. Suppose that $R^{*}-r<(1-r)\left(1-1 / \mathcal{R}_{0}\right)$. Then $1-R^{*}>(1-r) / \mathcal{R}_{0}=\bar{\gamma} / \bar{\beta}$. Since $R(t)$ is increasing, it follows that $R(t) \leq R^{*}$ for all $t \geq t_{0}$. Then

$$
\frac{d I}{d t}=\beta(t)(1-I-R) I-\gamma(t) I \geq \alpha(t) I-\beta(t) I^{2},
$$

where $\alpha(t)=\beta(t)\left(1-R^{*}\right)-\gamma(t)$. Moreover,

$$
\bar{\alpha}=\frac{1}{\tau} \int_{0}^{\tau} \alpha(t) d t=\bar{\beta}\left(1-R^{*}\right)-\bar{\gamma}>0
$$

Choose $\eta$ such that $0<\eta<\bar{\alpha} / \bar{\beta}$. Since $I(t) \rightarrow 0$ as $t \rightarrow+\infty$, one can find $t_{1}>t_{0}$ such that $0 \leq I(t) \leq \eta$ for all $t \geq t_{1}$. Now (6) implies that

$$
\frac{d I}{d t} \geq(\alpha(t)-\beta(t) \eta) I
$$

for all $t \geq t_{1}$. So $I(t) \geq I\left(t_{1}\right) \exp \left(\int_{t_{1}}^{t}(\alpha(u)-\beta(u) \eta) d u\right)$ for all $t \geq t_{1}$. Because of the choice of $\eta$, we obtain that $I(t) \rightarrow+\infty$ as $t \rightarrow+\infty$, which contradicts $I(t) \leq 1$. Therefore, $R^{*}-r \geq(1-r)\left(1-1 / \mathcal{R}_{0}\right)$.

\subsection{Periodic SEIR system}

Model and definition of $\mathcal{R}_{0}$. Consider the system

$\frac{d S}{d t}=-\beta(t) S I, \quad \frac{d E}{d t}=\beta(t) S I-\delta(t) E, \quad \frac{d I}{d t}=\delta(t) E-\gamma(t) I, \quad \frac{d R}{d t}=\gamma(t) I$,

with $S+E+I+R=1$ and where the rate $\delta(t)$ of moving from the latent compartment $E$ to the infectious compartment $I$ may also be $\tau$-periodic with $\bar{\delta}>0$. Consider the initial condition

$$
S\left(t_{0}\right)=1-e-i-r, \quad E\left(t_{0}\right)=e, \quad I\left(t_{0}\right)=i, \quad R\left(t_{0}\right)=r,
$$

with $e \geq 0, i \geq 0, r \geq 0, e+i>0$, and $e+i+r<1$. For any $\lambda>0$, let $\Phi\left(t, t_{0} ; \lambda\right)$ be the evolution operator associated with the $\tau$-periodic linear system

$$
\frac{d}{d t}\left(\begin{array}{c}
\tilde{E} \\
\tilde{I}
\end{array}\right)=\left(\begin{array}{cc}
-\delta(t) & \frac{\beta(t)(1-r)}{\lambda} \\
\delta(t) & -\gamma(t)
\end{array}\right)\left(\begin{array}{c}
\tilde{E} \\
\tilde{I}
\end{array}\right) .
$$

The spectral radius $\sigma(\lambda)$ of $\Phi\left(t_{0}+\tau, t_{0} ; \lambda\right)$ is called the dominant Floquet multiplier of (7) and does not depend on $t_{0}$. The off-diagonal rates of (7) being positive, [14, Lemma 2] implies that $\Phi\left(t, t_{0} ; \lambda\right)$ is a positive matrix for $t>t_{0}$. Moreover, $\sigma(\lambda)$ is a decreasing function of $\lambda[6]$. In $[4, \S 3.4]$ (see also [6]), the basic reproduction number $\mathcal{R}_{0}$ was defined as the unique $\lambda>0$ such that $\sigma(\lambda)=1$. 
Some remarks. It follows from $[2, \S A .1]$ that the periodic SEIR system has a unique solution defined for all $t \geq t_{0}$ and that $S(t)>0, E(t)>0$ and $I(t)>0$ for all $t>t_{0} . S(t)$ decreases and converges to $S^{*}$. $R(t)$ increases and converges to $R^{*}$. Since $\frac{d}{d t}(I+R)=\delta(t) E$, the function $I+R$ increases and converges. So $I(t) \rightarrow I^{*}$. Moreover, $R(t)-r=\int_{t_{0}}^{t} \gamma(u) I(u) d u$ converges as $t \rightarrow+\infty$. So $\bar{\gamma}>0$ implies that $I^{*}=0$. But $E=1-S-I-R$ shows that $E(t) \rightarrow E^{*}$. Since $\frac{d}{d t}(S+E)=-\delta(t) E$, the integral $\int_{t_{0}}^{\infty} \delta(u) E(u) d u$ converges. So $\bar{\delta}>0$ implies that $E^{*}=0$. Let us show that $S^{*}>0$. Imagine that $S^{*}=0$. Then

$$
\log S(t)-\log S\left(t_{0}\right)=-\int_{t_{0}}^{t} \beta(u) I(u) d u
$$

shows that $\int_{t_{0}}^{\infty} \beta(u) I(u) d u=+\infty$. But the inequalities

$$
\begin{aligned}
\int_{t_{0}}^{t} \beta(u) I(u) d u & \leq\left[\max _{0 \leq u \leq \tau} \frac{\beta(u)}{\gamma(u)}\right] \int_{t_{0}}^{t} \gamma(u) I(u) d u \\
\int_{t_{0}}^{t} \gamma(u) I(u) d u & =R(t)-r \leq 1-r
\end{aligned}
$$

show that $\int_{t_{0}}^{\infty} \beta(u) I(u) d u<+\infty$. Hence, $S^{*}>0$ and $R^{*}=1-S^{*}<1$.

Below the threshold. Since $S=1-E-I-R$, we have

$$
\frac{d}{d t}\left(\begin{array}{c}
E \\
I
\end{array}\right) \leq\left(\begin{array}{cc}
-\delta(t) & \beta(t)(1-r) \\
\delta(t) & -\gamma(t)
\end{array}\right)\left(\begin{array}{c}
E \\
I
\end{array}\right)
$$

where the inequality between vectors means inequality for each component. So $(E(t), I(t))^{\prime} \leq \Phi\left(t, t_{0} ; 1\right)(e, i)^{\prime}$, where the prime' stands for transposition. Assume that $\mathcal{R}_{0}<1$. Then $\sigma(1)<1$ and the matrix $\Phi\left(t, t_{0} ; 1\right)$ is bounded in norm by $K \exp \left(-\xi\left(t-t_{0}\right)\right)$ with $K>0$ and $\xi>0$ [15, Theor. 7.2]. So $R^{*}-r=\int_{0}^{\infty} \gamma(t) I(t) d t$ will tend to 0 if $e$ and $i$ tend to 0 .

Above the threshold. Assume $\mathcal{R}_{0}>1$. Imagine that the inequality $R^{*}-$ $r \geq(1-r)\left(1-1 / \mathcal{R}_{0}\right)$ is wrong. Then $1-R^{*}>(1-r) / \mathcal{R}_{0}$ and $\sigma((1-$ $\left.r) /\left(1-R^{*}\right)\right)>\sigma\left(R_{0}\right)=1$. By continuity of the spectral radius and because $R^{*}<1$, one can find $\eta>0$ such that $\eta<1-R^{*}$ and $\sigma(\lambda)>1$, where $\lambda=(1-r) /\left(1-R^{*}-\eta\right)$. We have $S(t) \rightarrow 1-R^{*}$ as $t \rightarrow+\infty$. So there is a $t_{1}>t_{0}$ such that $S(t) \geq 1-R^{*}-\eta$ for all $t \geq t_{1}$. It follows that

$$
\frac{d}{d t}\left(\begin{array}{c}
E \\
I
\end{array}\right) \geq\left(\begin{array}{cc}
-\delta(t) & \beta(t)\left(1-R^{*}-\eta\right) \\
\delta(t) & -\gamma(t)
\end{array}\right)\left(\begin{array}{c}
E \\
I
\end{array}\right)
$$

and $(E(t), I(t))^{\prime} \geq \Phi\left(t, t_{1} ; \lambda\right)\left(E\left(t_{1}\right), I\left(t_{1}\right)\right)^{\prime}$ for all $t \geq t_{1}$. In particular,

$$
\left(\begin{array}{c}
E\left(t_{1}+n \tau\right) \\
I\left(t_{1}+n \tau\right)
\end{array}\right) \geq \Phi\left(t_{1}+n \tau, t_{1} ; \lambda\right)\left(\begin{array}{c}
E\left(t_{1}\right) \\
I\left(t_{1}\right)
\end{array}\right)=\Phi\left(t_{1}+\tau, t_{1} ; \lambda\right)^{n}\left(\begin{array}{c}
E\left(t_{1}\right) \\
I\left(t_{1}\right)
\end{array}\right)
$$


for all integer $n \geq 1$. Let $\mu_{1}$ and $\mu_{2}$ be the eigenvalues of the positive matrix $\Phi\left(t_{1}+\tau, t_{1} ; \lambda\right)$, where $\mu_{1}=\sigma(\lambda)$ is the dominant eigenvalue of Perron-Frobenius theory [16]. Using Liouville's formula, we know that

$\operatorname{det}\left[\Phi\left(t_{1}+\tau, t_{1} ; \lambda\right)\right]=\mu_{1} \mu_{2}=\exp \left(-\int_{0}^{\tau}[\delta(t)+\gamma(t)] d t\right)=\exp (-(\bar{\delta}+\bar{\gamma}) \tau)<1$

Since $\mu_{1}=\sigma(\lambda)>1$, it follows that $\mu_{2}$ is real and $0<\mu_{2}<1$. Let $\left(p_{1,1}, p_{2,1}\right)^{\prime}$ be a positive eigenvector of the positive matrix $\Phi\left(t_{1}+\tau, t_{1} ; \lambda\right)$ associated with the eigenvalue $\mu_{1}$, as given by Perron-Frobenius theory. Let $\left(p_{1,2}, p_{2,2}\right)^{\prime}$ be a (real) eigenvector associated with $\mu_{2}$. As nonnegative eigenvectors can only be associated with $\mu_{1}\left[16\right.$, Theor. 2.1.4], it follows that $p_{1,2} p_{2,2}<0$. So one can assume that $p_{2,2}>0$ and $p_{1,2}<0$. Set

$$
P=\left(\begin{array}{ll}
p_{1,1} & p_{1,2} \\
p_{2,1} & p_{2,2}
\end{array}\right) .
$$

Then $\Phi\left(t_{1}+\tau, t_{1} ; \lambda\right)^{n}=P \operatorname{diag}\left(\mu_{1}^{n}, \mu_{2}^{n}\right) P^{-1}$ for all integer $n \geq 1$. Let $\Delta=$ $p_{1,1} p_{2,2}-p_{1,2} p_{2,1}>0$ be the determinant of $P$. Then

$$
\begin{gathered}
\left(\begin{array}{c}
E\left(t_{1}+n \tau\right) \\
I\left(t_{1}+n \tau\right)
\end{array}\right) \geq \frac{1}{\Delta}\left(\begin{array}{ll}
p_{1,1} & p_{1,2} \\
p_{2,1} & p_{2,2}
\end{array}\right)\left(\begin{array}{cc}
\mu_{1}^{n} & 0 \\
0 & \mu_{2}^{n}
\end{array}\right)\left(\begin{array}{cc}
p_{2,2} & -p_{1,2} \\
-p_{2,1} & p_{1,1}
\end{array}\right)\left(\begin{array}{c}
E\left(t_{1}\right) \\
I\left(t_{1}\right)
\end{array}\right) \\
=\frac{1}{\Delta}\left(\begin{array}{c}
\mu_{1}^{n} p_{1,1}\left[p_{2,2} E\left(t_{1}\right)-p_{1,2} I\left(t_{1}\right)\right]+\mu_{2}^{n} p_{1,2}\left[-p_{2,1} E\left(t_{1}\right)+p_{1,1} I\left(t_{1}\right)\right] \\
\mu_{1}^{n} p_{2,1}\left[p_{2,2} E\left(t_{1}\right)-p_{1,2} I\left(t_{1}\right)\right]+\mu_{2}^{n} p_{2,2}\left[-p_{2,1} E\left(t_{1}\right)+p_{1,1} I\left(t_{1}\right)\right]
\end{array}\right) .
\end{gathered}
$$

It follows from $\mu_{1}>1,0<\mu_{2}<1, \Delta>0, p_{1,1}>0, p_{2,1}>0$ and $p_{2,2} E\left(t_{1}\right)-$ $p_{1,2} I\left(t_{1}\right)>0$ that both $E\left(t_{1}+n \tau\right)$ and $I\left(t_{1}+n \tau\right)$ tend to $+\infty$ as $n \rightarrow+\infty$. But this contradicts $(E(t), I(t)) \rightarrow(0,0)$ as $t \rightarrow+\infty$. So $R^{*}-r \geq(1-r)\left(1-1 / \mathcal{R}_{0}\right)$.

\subsection{A periodic system describing vector-borne diseases}

Consider the system describing a vector-borne disease

$$
\frac{d S}{d t}=-\frac{\beta S J}{H}, \quad \frac{d I}{d t}=\frac{\beta S J}{H}-\gamma I, \quad \frac{d R}{d t}=\gamma I, \quad \frac{d J}{d t}=\beta^{\prime}(V(t)-J) I-\delta J,
$$

with a periodic vector population $V(t)$, and where $H$ is the total human population, $S+I+R=1, J$ is the number (not the fraction) of infected vectors, $\delta$ is the vector mortality, and $\beta$ (resp. $\beta^{\prime}$ ) is the vector biting rate multiplied by the transmission probability from vector to human (resp. from human to vector). This is reasonable model for arbovirus epidemics: dengue fever, West Nile fever, yellow fever, chikungunya. . The initial condition is $S\left(t_{0}\right)=1-i-r, I\left(t_{0}\right)=i$, $R\left(t_{0}\right)=r, J\left(t_{0}\right)=j$, with $i>0, r \geq 0, i+r<1$, and $0 \leq j \leq V\left(t_{0}\right)$. The basic reproduction number $\mathcal{R}_{0}$ is such that system

$$
\frac{d}{d t}\left(\begin{array}{c}
\tilde{I} \\
\tilde{J}
\end{array}\right)=\left(\begin{array}{cc}
-\gamma & \frac{\beta(1-r)}{\mathcal{R}_{0} H} \\
\beta^{\prime} V(t) & -\delta
\end{array}\right)\left(\begin{array}{c}
\tilde{I} \\
\tilde{J}
\end{array}\right)
$$


has a dominant Floquet multiplier equal to 1 [4] (some authors prefer to use $\left.\mathcal{R}_{0}^{\prime}=\sqrt{\mathcal{R}_{0}}\right)$. One can show as in $\S 3.2$ that the final size $R^{*}-r$ of the epidemic in humans tends to 0 when $\mathcal{R}_{0}<1$ and both $i$ and $j$ tend to 0 , and that $R^{*}-r \geq(1-r)\left(1-1 / \mathcal{R}_{0}\right)$ if $\mathcal{R}_{0}>1$. We briefly sketch the proof. When $\mathcal{R}_{0}<1$, the result follows from the fact that

$$
\frac{d}{d t}\left(\begin{array}{c}
I \\
J
\end{array}\right) \leq\left(\begin{array}{cc}
-\gamma & \frac{\beta(1-r)}{H} \\
\beta^{\prime} V(t) & -\delta
\end{array}\right)\left(\begin{array}{l}
I \\
J
\end{array}\right) .
$$

When $\mathcal{R}_{0}>1$, we have $R(t) \rightarrow R^{*}, S(t) \rightarrow 1-R^{*}, I(t) \rightarrow 0$, and $J(t) \rightarrow 0$ as $t \rightarrow+\infty$. Assume that $1-R^{*}>(1-r) / \mathcal{R}_{0}$. Then one can find $\eta>0$ and $t_{1}>t_{0}$ such that

$$
\frac{d}{d t}\left(\begin{array}{l}
I \\
J
\end{array}\right) \geq\left(\begin{array}{cc}
-\gamma & \frac{\beta\left(1-R^{*}-\eta\right)}{H} \\
\beta^{\prime}(V(t)-\eta) & -\delta
\end{array}\right)\left(\begin{array}{l}
I \\
J
\end{array}\right) .
$$

for all $t \geq t_{1}$, the dominant Floquet multiplier of the right side being strictly bigger than 1. This leads as in $\S 3.2$ to a contradiction with $I(t) \leq 1$. So $1-R^{*} \leq(1-r) / \mathcal{R}_{0}$.

\section{Conclusion}

Our analysis shows that the threshold theorem for systems with constant coefficients (with the two classical cases, $\mathcal{R}_{0}<1$ and $\mathcal{R}_{0}>1$ ) can be generalized to systems with periodic coefficients representing seasonality, provided the basic reproduction number $\mathcal{R}_{0}$ is defined as in our earlier studies $[3,4,5]$. However, somewhat unexpectedly, periodic systems may have relatively large epidemics even when $\mathcal{R}_{0}<1$ and the final epidemic size may not increase with $\mathcal{R}_{0}$ or the initial fraction $i$ of infected people.

These observations based on simple systems should serve as a warning for the interpretation of epidemics influenced by seasonality. Epidemics of emerging vector-borne diseases, which receive a renewed attention because of climate change theory, should be analyzed with caution as we have shown with the case of chikungunya in Réunion and Italy. Another case of interest nowadays is that of pandemic flu in humans following bird flu. The pandemic of 1918-1919 occured in several waves influenced by seasonality. Attempts to estimate the basic reproduction number for this pandemic have assumed constant coefficients and have used the beginning of the epidemic curve or the final size of singlewave epidemics (see, e.g., [17]). Our work suggests that these analyses may have to be revised since the relationship between $\mathcal{R}_{0}$ and the behavior of epidemics influenced by seasonality is not a straightforward generalization of what is known for the case of a constant environment.

\section{Acknowledgment}

This work was initiated while N.B. was visiting Instituto Gulbenkian de Ciência, with funding from the Portuguese Research Council (FCT) and the European 
Commission (grant MEXT-CT-2004-14338). N.B. also thanks the European $\mathrm{CDC}$ for its invitation to a chikungunya modeling meeting.

\section{References}

[1] W. O. Kermack, A. G. McKendrick, (1927), A contribution to the mathematical theory of epidemics, Proc. Roy. Soc. 115A, 700-721.

[2] H. R. Thieme (2003) Mathematics in Population Biology, Princeton University Press, Princeton.

[3] N. Bacaër, S. Guernaoui, (2006), The epidemic threshold of vector-borne diseases with seasonality, J. Math. Biol. 53, 421-436.

[4] N. Bacaër, (2007), Approximation of the basic reproduction number $R_{0}$ for vector-borne diseases with a periodic vector population, Bull. Math. Biol. 69, 1067-1091.

[5] N. Bacaër, R. Ouifki, (2007), Growth rate and basic reproduction number for population models with a simple periodic factor, Math. Biosci. 210, 647-658.

[6] W. Wang, X.-Q. Zhao, (2008), Threshold dynamics for compartmental epidemic models in periodic environments, J. Dyn. Diff. Equat. 20, 699-717.

[7] N. Bacaër, Periodic matrix population models: growth rate, basic reproduction number and entropy. Submitted.

[8] O. Diekmann, J. A. P. Heesterbeek (2000) Mathematical Epidemiology of Infectious Diseases, Wiley, Chichester.

[9] European Centre for Disease Prevention and Control (2009) Report, Expert Meeting on Chikungunya Modelling, ECDC, Stockholm.

[10] R. M. Anderson, R. M. May (1991) Infectious Diseases of Humans: Dynamics and Control, Oxford University Press, Oxford.

[11] J. Ma, D. J. D. Earn, (2006), Generality of the final size formula for an epidemic of a newly invading infectious disease, Bull. Math. Biol. 68, 679702 .

[12] J. Arino, F. Brauer, P. van den Driessche, J. Watmough, J. Wu, (2007), A final size relation for epidemic models, Math. Biosci. Eng. 4, 159-175.

[13] N. Bacaër, X. Abdurahman, (2008), Resonance of the epidemic threshold in a periodic environment, J. Math. Biol. 57, 649-673.

[14] G. Aronsson, R. B. Kellogg, (1978), On a differential equation arising from compartmental analysis, Math. Biosci. 38, 113-122. 
[15] J. K. Hale (1980) Ordinary Differential Equations, Krieger, Malabar.

[16] A. Berman, R. J. Plemmons, (1979), Nonnegative Matrices in the Mathematical Sciences, Academic Press, New York.

[17] E. Vynnycky, A. Trindall, P. Mangtani, (2007), Estimates of the reproduction numbers of Spanish influenza using morbidity data, Int. J. Epidem. $36,881-889$. 
$$
\text { *Tex version of revised paper (LaTeX or Word) unmarked copy }
$$

Click here to download Tex version of revised paper (LaTeX or Word) unmarked copy: BacaerGomesFinal.tex 